\title{
EIGENTUMS-, BESCHAFTIGUNGS- UND PRODUKTIONSVERANDERUNGEN IN DER PERUANISCHEN LANDWIRTSCHAFT DURCH DIE AGRARREFORM VON 1969
}

\author{
Von Mechthild Minkner
}

\section{Einführung}

Im März 1976 stellt Ernest Feder, internationaler Agrarexperte, spezialisiert in Fragen der Agrarstruktur und der ländlichen Entwicklung Lateinamerikas, zur peruanischen Agrarreform fest: „Obwohl Ursprung, Wesen, Ausmaß und Folgen der peruanischen Agrarreform komplexe Sachverhalte darstellen und obwohl ein beträchtlicher Bereich der peruanischen Landwirtschaft bedeutende strukturelle Anderungen erfahren hat, nachdem ein Teil der traditionellen Landelite Land und Macht verloren hatte, ist die Masse der Landbevölkerung meiner Ansicht nach wieder benachteiligt worden ... “". Nach Ansicht Feders sind die Bauern politisch hintergangen worden, weil die für die Agrarreform verantwortlich zeichnenden Militärs niemals die Absicht gehabt hätten, ihnen weiterreichende Partizipationsmöglichkeiten in wirtschaftlichen, sozialen und politischen Entscheidungsprozessen auf lokaler, regionaler und nationaler Ebene einzuräumen. Für die wirtschaftliche Benachteiligung der Mehrzahl der landlosen und landarmen Bevölkerung führt er als Argumente an: a) Die Zahl der von der Agrarreform begünstigten Personen ist gering. b) Die landwirtschaftliche Produktion zeigt seit 1969 kein nennenswertes Wachstum, so daß bei steigenden Preisen und stagnierendem Nominaleinkommen das Realeinkommen gefallen ist und damit eine weitere Verarmung bei einem großen Teil der Kleinstbauern und Landarbeiter stattgefunden hat. c) Die anfängliche Priorität des Agrarsektors im Reformprozeß wurde sehr bald zu Gunsten der Förderung anderer Sektoren abgebaut.

Gemäß ihrer gesetzlichen Grundlage (D. L. $17716^{2}$ ) und den politischen Verlautbarungen der Velasco-Regierung ${ }^{3}$ ist die Agrarreform ein integraler Prozeß und ein Instrument zur Transformation und Neuordnung der Agrarstruktur, um die marginalisierten ländlichen Bevölkerungsgruppen in den nationalen Entwicklungsprozeß einzugliedern und über eine Dynamisierung der Landwirtschaft und die Reorientierung finanzieller Ressourcen die Industrialisierung des Landes zu fördern. Damit geht sie ihrem gesetzlich verankerten und propagierten Anspruch nach über eine Landreform hinaus. Art. 1 D. L. 17716 postuliert denn auch, daß Latifundismus und Minifundismus durch sozial gerechte Eigentums-, Besitz- und Arbeitsverhältnisse ersetzt werden sollen, um einerseits Einkommens- und Entwicklungsniveau der ländlichen Bevölkerung anzuheben, andererseits Produktion

\footnotetext{
1 Feder, Ernest, Vorwort zu Gaitzsch, Andrea, Die peruanische Agrarreform - Landgemeinden versus Genossenschaften, Die Dritte Welt, Sonderheft 1976, Meisenheim am Glan 1976, o. S. Die anschließenden sinngemäßen Übernahmen im ersten Absatz stammen aus derselben Quelle.

2 D. L. 17716 vom 24. 6. 1969 wurde im Verlaufe der Agrarreform durch eine Vielzahl von gesetzlichen Bestimmungen ergänzt, um den praktischen und politischen Erfordernissen der. Durchführung gerecht zu werden. Vgl. dazu die Gesetzessammlung von Dongo Denegri, L., Compendio Agrario, Tomo III, Cuzco 1974; Tomo IV, Lima 1976.

3 Vgl. Velasco - La voz de la revolución, Discursos del Presidente de la República General de División Juan Velasco Alvarado 1968-1970, Lima o. J., S. 43 ff.
} 
und Produktivität im Agrarsektor zu erhöhen. Kern der Agrarreformmaßnahmen sind die Enteignung der landwirtschaftlichen Großbetriebe ${ }^{4}$ und deren Umwandlung prioritär in gemeinschaftliche Produktionsformen wie Genossenschaften. Die Transformation der Eigentums- und Besitzverhältnisse, Grundlage und Reflex der Machtbeziehungen in der Nutzung und Bewirtschaftung des Bodens, verlangt eine Neustrukturierung des landwirtschaftlichen Unternehmens in seiner technischen, ökonomischen und sozialen Dimension unter Berücksichtigung der unterschiedlichen Formen der Arbeitsorganisation und des sozialen Beziehungsgeflechts der am Produktionsprozeß beteiligten Personen ${ }^{5}$. Nach Art. 2 und 3 D. L. 17716 sollen Eigentums- und Besitzreform mit einer Neuausrichtung der Kredit-, Absatzund Beschaffungsmechanismen, der Preisbildung, der Erforschung und Erschließung der natürlichen Ressourcen sowie der Verbesserung der Infrastruktur im weitesten Sinn für den Agrarsektor resp. die ländlichen Gebiete verbunden werden. Tiefergehende strukturelle Veränderungen im Agrarbereich verlangen Reformen in der gesamten Gesellschaftsstruktur, vor allem aber im Subsystem Wirtschaft. Sie machen eine globale und sektorale Planung mit obligatorischem und nicht mit indikativem Charakter notwendig ${ }^{6}$.

Am 7. Jahrestag der Verkündung des Agrarreformgesetzes stellte der damalige Chef der Dirección General de Reforma Agraria y Asentamiento Rural (DGRAAR) in einem Interview fest, daß über Reichweite und Auswirkungen der Agrarreform und der sog. empresas asociativas ${ }^{7}$, über ihre Erfolge, Probleme und Perspektiven, sei es im ökonomischen, sozialen, kulturellen und politischen Bereich, auf Makround auf Mikroebene keine umfassendere und kohärente sowie methodisch und empirisch abgesicherte Evaluierung existiert ${ }^{8}$. Bis heute muß die Agrarreform auf der Grundlage der Kenntnis einzelner Problembereiche und Unternehmen, gewonnen in der Durchführungspraxis, beurteilt werden. Hinzu kommen von akademischer Seite einige Case Studies ${ }^{9}$ sowie mehrere globale, stärker politisch orientierte Abhandlungen ${ }^{10}$. Im Rahmen der Seminararbeit des Centro de Participación Popular11 entstand 1974 eine Reihe von Beschreibungen und Analysen von im Rahmen der Agrarreform geschaffenen Unternehmen. Eine quantitative und qualitative Untersuchung der Agrarreform und der neuen Unternehmen, vor allem ihrer Implikationen im sozioökonomischen Bereich auf Makroebene, wurde einerseits aufgrund der aufwendigen empirischen Arbeit und der methodischen Schwierigkeiten zurückgestellt; andererseits dürften neben personellen und finanziellen

4 Eine Darstellung der Enteignungsmodalitäten von D. L. 17716 findet sich z. B. bei v. Urff, W., Die peruanische Agrarreform, in: Zeitschrift für ausländische Landwirtschaft, Jg. 14, H. 4, 1975, S. $331 \mathrm{ff}$. , sowie bei Wyss, O., Wandel im Ordnungsgefüge der peruanischen Wirtschaft unter der Revolutionsregierung 1968-1974, Bern und Stuttgart 1976, S. $39 \mathrm{ff}$.

5 Vgl. zum Begriff der Besitz- und Arbeitsverhältnisse, der sog. tenencia de la tierra, Barraclough, S. und J. C. Collarte, El hombre y la tierra en América Latina, Resumen de los Informes CIDA sobre tenencia de la tierra en Argentina, Brasil, Colombia, Chile, Ecuador, Guatemala, Perú, Santiago de Chile 1972, S. 12 .

6 Valdez Angulo, E., El sector agrario - planteamientos y acciones del Gobierno Revolucionario de la Fuerza Armada, Exposición en el CAEM, 16 de mayo de 1974, Lima o. J., S. 11.

7 Der spanische Begriff "empresas asociativas" wird in diesem Artikel mit "gemeinschaftliche Produktionsformen" übersetzt.

8 Tres preguntas al Director de Reforma Agraria, in: Marka, 24. 6. 1976, S. 29.

9 Die beiden wichtigsten deutschsprachigen Untersuchungen sind von Kressin, J. und Erich Spiegel, Agrarreform und Produktionsgenossenschaften in Peru, Lateinamerika-Institut der Freien Universität Berlin, Materialien zur Lehre und Forschung, Nr. 1, Berlin 1973, und Gaitzsch, Andrea, Die peruanische Agrarreform - Landgemeinden versus Genossenschaften, Die Dritte Welt, Sonderheft 1976, Meisenheim am Glan 1976.

10 Eine Abhandlung neueren Datums stammt von Valderrama, M., Siete años de Reforma Agraria Peruana 1969-1976, Lima 1976.

11 Die mehr als 50 Kurzstudien sind Ergebnis der Feldarbeit der Seminarteilnehmer. 
Engpässen im Ministerio de Agricultura auch politische „Empfindlichkeiten“ eine Rolle gespielt haben.

Auf dem Hintergrund des Kommentars von Feder einerseits und der zum Teil noch herrschenden Ansicht über die große Reichweite der peruanischen Agrarreform andererseits wird in diesem Artikel versucht, einige Aspekte, die im Zusammenhang mit Umfang und Auswirkungen der Agrarreform vor allem auf makroökonomischer Ebene stehen, aufzuzeigen. Die vorher angedeutete sehr lückenhafte Materiallage erlaubt allerdings nur grobrastrige, tendenzielle Aussagen. Außerdem ist der Zeitpunkt für eine endgültige Beurteilung noch zu früh. Materialbasis für die nachfolgenden Ausführungen sind vor allem Sekundärliteratur sowie Informationen aus Gesprächen mit Vertretern der für den Agrarsektor zuständigen Institutionen und der neuen Unternehmen.

\section{Umfang und Auswirkungen der Agrarreform}

Oberstes instrumentales Ziel der Agrarreform ist die Transformation des Latifundien-/Minifundiensystems. Im Einklang damit zielt die erste Etappe der Agrarreform darauf $\mathrm{ab}$, alle Produktionseinheiten $\mathrm{zu}$ enteignen, die nicht persönlich bewirtschaftet werden sowie von den persönlich geführten Produktionseinheiten diejenigen, die über den von D. L. 17716 festgelegten Maximalgrenzen an Boden für kleines bis mittleres Eigentum liegen. Die Größe der persönlich bewirtschafteten Flächen, die nicht unter die Agrarreform fallen, variiert entsprechend der Region (Costa, Sierra, Ceja de la Selva), ob es sich um bewässertes oder unbewässertes Land oder natürliche Weiden handelt ${ }^{12}$.

Tabelle 1 Höchstgrenzen des nicht unter die Agrarreform fallenden persönlich bewirtschafteten Eigentums (in ha):

\begin{tabular}{lccc}
\hline \multirow{2}{*}{ Region } & \multicolumn{2}{c}{ Ackerland } & Naturweiden \\
\hline Costa & bewässert & unbewässert & \\
& 150 & $-*$ & 1.500 \\
Sierra und Ceja de la Selva*** & $(200)$ & & $(4 \mathrm{mal})$ \\
& $15-55$ & $30-110$ & $\mathbf{-}^{* * *}$ \\
& $(45-165)$ & $(90-330)$ & $(4 \mathrm{mal})$ \\
\hline
\end{tabular}

* Vom Gesetz nicht angegeben, da in der Costa praktisch nur bewässertes Land bebaut werden kann. Analog zur Regelung für die Sierra würde die Höchstgrenze bei 300 ha liegen.

** In der Sierra und Ceja de la Selva variieren die Höchstgrenzen nach Provinzen.

:** Fläche, die zum Halten von 5000 Schafeinheiten erforderlich ist.

Anmerkung: Die Zahlen in Klammern geben die möglichen Verschiebungen der Höchstgrenzen an (vgl. Fußnote 12).

Quelle: Art. 28 bis 31 D. L. 17716.

12 Bei Vorliegen bestimmter produktionstechnischer, arbeitsrechtlicher und steuerrechtlicher Voraussetzungen können die Höchstgrenzen verschoben werden (vgl. dazu D. L. 17716 Art. 34). 
D. L. 21333 in Verbindung mit D. S. 173-76-AG bestimmt ab 10. 12. 1975 neue Höchstgrenzen der nicht unter die Agrarreform fallenden Unternehmen und damit des kleinen bis mittleren Eigentums. Die Maximalgröße für einen Mittelbetrieb an der Küste wird auf 50 ha Bewässerungsland, in der Sierra und Ceja de la Selva auf 30 ha herabgesetzt. Für unbewässertes Land gilt die doppelte Fläche. Direkt geführte Viehzuchtbetriebe dürfen so viel Weideland besitzen, wie zur Haltung von 3000 Schafeinheiten notwendig ist. Das Änderungsgesetz kommt rückwirkend nur dann zur Anwendung, wenn das Landaussonderungsverfahren (procedimiento de afectación) bis zum Inkrafttreten des Gesetzes noch nicht abgeschlossen war ${ }^{13}$. Das enteignete Land geht in Eigentum der DGRAAR über, um an landlose und landarme Bauern bzw. Landarbeiter übereignet zu werden, und zwar prioritär an in Produktionsgenossenschaften (CAP), Sociedades Agrícolas de Interés Social (SAIS) und Comunidades Campesinas zusammengeschlossenen Nutznießer der Agrarreform (Art. 67 D. L. 17 716). Die von der Agrarreform begünstigten Personen und Unternehmen schließen mit dem Staat einen Kaufvertrag ab. Bis zur Begleichung der Agrarschuld (20 Jahre; bis zu 5 Freijahre) besteht ein Eigentumsvorbehalt. Zur Lösung des Minifundienproblems sieht das Gesetz ein Teilungsverbot für Kleinstbesitz vor, definiert als die sog. unidad agrícola familiar mit mindestens 3 ha (Art. 98 D. L. 17 716). Der Parzellierung und Bodenaufsplitterung soll durch Eigentumszusammenlegungen und Flurbereinigung entgegengewirkt werden (Art. 99 und 102 D. L. 17 716).

\section{Auswirkungen auf die Eigentums- und Besitzstruktur}

a) Stand der Enteignungen und Zuweisungen

Gemäß Schätzungen der DGRAAR sollten die Enteignungen (Planziel 12,6 Millionen ha ${ }^{14}$ ) Ende 1975 abgeschlossen sein. Aufgrund der komplizierten administrativen Abwicklung [durchschnittlich zwei Jahre für die vier Hauptetappen: Landaussonderung (afectación), Bewertung (valorización), Enteignung (expropiación), Zuweisung (adjudicación)] und damit der tatsächlichen Fortschritte der Agrarreform sowie des vom Staat aufzubringenden Finanzvolumens wurde schon 1972 der Abschluß der Enteignungen auf Ende 1976 und der Abschluß der Zuweisungen auf 1978 verschoben. Die enteigenbare Fläche wurde mit 10,2 Millionen ha und 14500 sog. predios ebenfalls neu geschätzt ${ }^{15}$. Stand und Entwicklung der Enteignungen, Entschädigungen und Zuweisungen sind aus den Tabellen 2 bis $5 \mathrm{zu}$ entnehmen.

\footnotetext{
13 Dongo Denegri stellt fest, daß es wohl nur in wenigen Fällen zu einer rückwirkenden Anwendung kommen würde (vgl. Dongo Denegri, L., Compendio Agrario, Tomo IV, a. a. O., S. 191).

14 Oficina Sectorial de Planificación, Ministerio de Agricultura, Reforma Agraria - Perú, Lima 1972, Statistischer Anhang, Tabelle 1.

15 Oficina Sectorial de Planificación, Ministerio de Agricultura, Evaluación del Plan de Desarrollo del Sector Agrario, Lima o. J., Statistischer Anhang, Tabelle 2.
} 
Tabelle 2 Enteignungen von 1963 bis 1976

\begin{tabular}{lrrrr}
\hline & Betriebe & \multicolumn{1}{c}{$\begin{array}{c}\text { Fläche } \\
\text { (in ha) }\end{array}$} & $\begin{array}{c}\text { Vieh } \\
\text { (Stückzahl) }\end{array}$ & $\begin{array}{r}\text { Entschädigung } \\
\text { (in 1000 S/.) }\end{array}$ \\
\hline 1963-1970 & 1186 & 3077668 & 1089293 & 6306042 \\
1971 & 425 & 617358 & 345321 & 1111869 \\
1972 & 1128 & 1016527 & 230476 & 2041306 \\
1973-1974 & 4625 & 1780156 & 163847 & 2446127 \\
1975 & 2376 & 933919 & 205861 & 1254834 \\
30. 6. 1976 & 1370 & 406371 & 22872 & 610421 \\
1963-1976 & 11110 & 7831999 & 2057670 & 13770599 \\
$\begin{array}{l}\text { (30. 6. 76) } \\
\text { Geplantes Volumen }\end{array}$ & & & & \\
Ende 1976 & 14500 & 10150000 & $-*$ & \\
\hline
\end{tabular}

$\because$ Keine Angaben.

Quelle: Verschiedene Nummern Evaluierungsberichte der Oficina Sectorial de Planificación, Ministerio de Agricultura.

Tabelle 3 Entwicklung der Enteignungen und Zuweisungen (1963 bis 1976 akkumuliert)

\begin{tabular}{lccc}
\hline & $\begin{array}{r}\text { Enteignungen } \\
\text { (in 1000 ha) }\end{array}$ & $\begin{array}{c}\text { Zuweisungen } \\
\text { (in 1000 ha) }\end{array}$ & $\begin{array}{c}\text { Zuweisungen zu } \\
\text { Enteignungen } \\
\text { (in \%) }\end{array}$ \\
\hline 1964-1969* & 1470 & 570 & 38,8 \\
(D. L. 15 037) & 3078 & 1280 & 41,6 \\
1970 (Dez.) & 3695 & 1838 & 49,7 \\
1971 (Dez.) & 4712 & 2958 & 62,8 \\
1972 (Dez.) & 5508 & 3832 & 69,6 \\
1973 (Aug.) & 6492 & 5144 & 79,2 \\
1974 (Dez.) & 7426 & 6227 & 83,9 \\
1975 (Dez.) & 7832 & $6803^{* *}$ & 86,9 \\
1976 (Juni) & & & \\
\hline
\end{tabular}

* Agrarreform der Regierung Belaúnde T. vom 21. 5. 1964.

* Enthält 172913 ha als Zuweisungen an Propiedad Social in den Agrarzonen IV und XII.

Quellen: Für 1970_1976 (außer August 1973) verschiedene Nummern der Evaluierungsberichte OSPA, Ministerio de Agricultura; August 1973: Horton, D. Land Reform und Reform Enterprises in Peru, Madison, June 1974, S. 83.

Zusammenfassend läßt sich zum Verlauf der Agrarreform anhand der Entwicklung der Enteignungen und Zuweisungen feststellen:

- Der Durchführungsrhythmus der Agrarreform ist im Zeitraum 1969 bis 1976 unterschiedlich gewesen. Man setzte bei den agroindustriellen Komplexen der 
Küste und den großen Viehhazienden in den durch Bauernunruhen politisch angespannten Gebieten der Sierra an. Bis Ende 1970 wurden mit 391 sog. fundos (Güter) und rund 1,6 Millionen ha etwa 15,8\% der vorgesehenen Fläche enteignet. Das sind in 11/2 Jahren der Anwendung von D. L. 17716 etwa die doppelte Hektarfläche im Vergleich zum Agrarreformgesetz Nr. 15037 unter Belaúnde. Mit abnehmender Fläche der „fundos“, aber gleichem administrativem Aufwand, mit wachsendem Widerstand der Eigentümer und unterschiedlichem „Komplicentum “ eines Teils der Regierungsfunktionäre ${ }^{16}$ verlangsamte sich der Rhythmus 1971. Landarbeiter und Bauern, vor allem der Küstentäler, begannen mit ihren Interessenvertretungen organisiert Druck auf die Abwicklung der Enteignungen und Übergaben auszuüben. Die Regierung verstärkte die Kapazität vor allem in den Agrarzonen und steigerte 1973/ 74 entscheidend die Haushaltszuweisungen für den Agrarsektor ${ }^{17}$. Bis Ende 1974 konnten mit 7364 „fundos“ und 6,5 Millionen ha die Enteignungen zwar wesentlich beschleunigt werden; jedoch ist die Agrarreform mit 50\% resp. $63 \%$ der geplanten Enteignungen stark im Verzug. Ab Mitte 1975 wurden die Enteignungen vor allem durch Finanzierungsschwierigkeiten bei sich rapide verschlechternder Haushaltslage, durch Anderungen in der Agrarreformgesetzgebung und durch sich anbahnenden politischen Kurswechsel behindert. Ab Mitte 1976 stagnierte die Agrarreform. Von dem Ziel, bis zum 31. 12.1976 14500 „fundos" mit 10,1 Millionen ha zu enteignen, konnten bis Ende Juni 1976 nur $76,6 \%$ resp. $72,2 \%$ erfüllt werden.

- Ein Vergleich der Enteignungen und Zuweisungen zeigt, daß zwar ab 1972 die Zuweisungen p. a. absolut und relativ gestiegen sind; jedoch lag die zugewiesene Fläche im Juni 1976 noch immer um 13\% (rund 1 Million ha) unter der enteigneten Fläche. Das Tempo der Zuweisungen hängt vom Auswahlprozeß der begünstigten Personen, von der Organisierung der neuen Unternehmen und der Ablösung der vorläufigen staatlichen Verwaltungskomitees durch die Selbstverwaltung der Mitglieder der sog. empresas asociativas ab. Die langwierige Abwicklung von der Landaussonderung bis zur Landzuweisung führt zu Dekapitalisierung und Produktionsrückgang, zur Vernachlässigung der Kulturen und der Nutzfläche, wodurch sich die Ertragskraft der Mehrzahl der Unternehmen vermindert hat. Dieser negative Prozeß setzte sich zum Teil noch unter den vorläufigen Verwaltungskomitees fort.

16 Vgl. Eguren, Fernando, Reforma Agraria, cooperativización y luchas campesinas. El Valle ChancayHuaral, DESCO, Lima 1975, S. 61.

17 1970/71 betrugen die Investitionen der öffentlichen Hand im Agrarsektor $13 \%$ der Gesamtinvestitionen. Sie erhöhten sich 1973/74 mit rund 7,3 Milliarden S/. (Durchschnittskurs des S/. 1973/74: 1 US= 43,38 S/.) auf fast ein Drittel der öffentlichen Investitionen (vgl. Banco Central de Reserva del Perú, Memoria 1973, S. 45; Memoria 1974, S. 41). 
Tabelle 4 Landzuweisungen nach Unternehmensformen (akkumuliert 1963 bis 1976)

\begin{tabular}{llcl}
\hline Unternehmensform & Anzahl & $\begin{array}{c}\text { Fläche } \\
(1000 \mathrm{ha})\end{array}$ & Familien \\
\hline
\end{tabular}

1972 (Juni)

Genossenschaften*

SAIS

134

811

44106

$10 \quad 800$

11691

Comunidades

61

331

15815

Einzelbetriebe

$-\frac{120}{2062}$

16367

87979

1973 (Dezember)

Genossenschaften*

SAIS

458

1878

88532

$39 \quad 1895$

39817

Comunidades

94

386

30360

Einzelbetriebe

- $\quad \frac{121}{4280}$

16727

175436

1974 (September)

Genossenschaften*

SAIS

547

2146

97584

$41 \quad 1964$

49878

Comunidades

111

439

34316

Einzelbetriebe

-**

17292

199070

1975 (Dezember)

Genossenschaften"

SAIS

973

3010

124820

56

2513

59353

Comunidades

180

51116

Einzelbetriebe

-**

18723

$\frac{136}{6228}$

254012

1976 (Juni)

Genossenschaften*

- **

3251

132384

SAIS

- $^{n * *}$

2592

59764

Comunidades

-"*

650

66237

Einzelbetriebe

$-* *$

$\frac{137}{6630^{* * * *}}$

19030

277415

\footnotetext{
* Einschließlich der sogenannten Grupos Campesinos.

** Keine Angaben.
}

*** Ohne Zuweisungen (172 913 ha) an Propiedad Social.

Quellen: Horton, D., Haciendas and Cooperatives, Madison, Sept. 73, S. 61 (1972); DGRAAR, Reforma Agraria en Cifras, Enero 74, Tab. 2 (1973); v. Urff, Die peruanische Agrarreform, a. a. O., S. 346/47; OSPA, Evaluierungsberichte (1975 und 1976). 
- Tabelle 4 läßt klar erkennen, daß die gesetzlich bestimmte Priorität der kooperativen und präkooperativen Produktionsformen bei der Zuweisung in der Praxis durchgesetzt wurde. CAP und SAIS überwiegen mit fast $70 \%$, gefolgt von den Comunidades Campesinas $(24 \%)$ und den bäuerlichen Einzelwirtschaften $(6 \%)$. Verzögerungen traten bei der Zuweisung von Land an die Comunidades ein, die bis Ende Juni 1976 nur 9,8\% der zugeteilten Fläche erhalten hatten. Ihr Sollanteil liegt bei 15,5\% der Fläche und 17,4\% der begünstigten Familien. Das bedeutet, daß in den Comunidades bis Juni 1976 mehr Familien $(41 \%)$ als geplant weniger Land $(37 \%)$ zugewiesen wurde. Auch bei den Einzelbetrieben konnte bis Juni 1976 noch nicht der Sollwert (3,9\% der insgesamt überwiesenen Fläche) erreicht werden ${ }^{18}$.

- In der Verteilung der Landzuweisungen nach natürlichen Regionen dominiert die Sierra (Tabelle 5). $81 \%$ der bis März 1976 zugeteilten Fläche lagen in der Sierra und Ceja de la Selva. Das Verteilungsmuster nach Unternehmensformen unterstreicht außerdem, daß die SAIS eine für die Sierra geschaffene spezielle vorgenossenschaftliche Form ist, mittels derer die um eine ehemalige Viehhazienda liegenden Comunidades über die Gewinnbeteiligung gefördert werden sollen, ohne daß die technisch-ökonomische Einheit des Unternehmens zerschlagen wird. Die unterschiedliche Relation Fläche zu begünstigten Personen an der Küste und in der Sierra spiegelt die stark unterschiedliche Qualität und Produktivität der Böden resp. der Unternehmen wider.

$18 \mathrm{Zu}$ den Sollwerten nach Unternehmensformen vgl. Horton, D., Land Reform and Reform Enterprises in Peru, Madison, June 1974, S. 79. 
Tabelle 5 Landzuweisungen nach Unternehmensformen und Regionen zum 31. 3. 1976

\begin{tabular}{|c|c|c|c|}
\hline Unternehmensform & Fläche (in ha) & Unternehmen & Familien \\
\hline \multicolumn{4}{|l|}{ Costa } \\
\hline Genossenschaften* & 796894 & 380 & 71883 \\
\hline SAIS & 5130 & 1 & 81 \\
\hline Comunidades Campesinas & 39553 & 10 & 9675 \\
\hline \multirow[t]{2}{*}{ Einzelbetriebe } & 67124 & & 10451 \\
\hline & 908701 & & 92090 \\
\hline \multicolumn{4}{|l|}{ Sierra, Ceja Selva } \\
\hline Genossenschaften* & 2255742 & 556 & 56442 \\
\hline SAIS & 2538479 & 56 & 59536 \\
\hline Comunidades Campesinas & 548552 & 179 & 44688 \\
\hline \multirow[t]{2}{*}{ Einzelbetriebe } & 94685 & & 9907 \\
\hline & 5437458 & & 170573 \\
\hline \multicolumn{4}{|l|}{ Selva } \\
\hline Genossenschaften* & 61597 & 43 & 1361 \\
\hline SAIS & 26678 & $1^{* *}$ & K. A.*** \\
\hline Comunidades Campesinas & - & - & - \\
\hline \multirow[t]{2}{*}{ Einzelbetriebe } & 305469 & & 13174 \\
\hline & 399744 & & $\overline{14535}$ \\
\hline
\end{tabular}

* Einschließlich der sog. Grupos Campesinos.

* Zweigbetrieb der SAIS Tupac Amaru, die in der Agrarzone X liegt. *** Keine Angaben.

Quelle: Ministerio de Alimentación, Dirección General de Informática y Estadística, Centro de Información, Vademecum Nr. 1 CI.

b) Veränderungen in der Eigentums- und Besitzstruktur

Kernproblem der ländlichen Entwicklung in Peru und damit Hauptgrund für die Agrarreform ist einerseits die extreme Größen- und Eigentumskonzentration, andererseits die exzessive Zersplitterung der Betriebsstruktur. Gemäß Zensus von $1961^{19}$ besaßen 0,1\% der Produktionseinheiten $61 \%$ (10,7 Millionen ha) der Nutzfläche; $0,4 \%$ der Betriebe (500 ha und mehr) $75 \%$. Das andere Extrem sind die Produktionseinheiten unter 5 ha, die mit $83 \%$ aller Einheiten nur 5,5\% der Gesamtfläche bewirtschafteten. Über $55 \%$ der Einheiten hatten nur bis zu 2 ha Eigentum; $35 \%$ nur bis zu 1 ha. Vor der Agrarreform dominierte das private Eigentum mit rund $74 \%$ aller Einheiten. Davon besaßen die Handelsgesellschaften (vielfach in Familienbesitz oder mit wenigen Teilhabern) mit 0,1\% der Einheiten allein 16,4\% der Flä-

19 Instituto Nacional de Planificación, Dirección Nacional de Estadística y Censos, Primer Censo Nacional Agropecuario, Resultados Finales, Lima 1961, S. 2, S. 12 bis 14. 
che. In bäuerlichem Einzelbesitz (68\% der Einheiten) waren $68 \%$ der Fläche. Diese war wiederum extrem ungleich verteilt; denn $83 \%$ waren mit weniger als 5 ha Eigentümer von $8 \%$ der Fläche. Die restlichen $17 \%$ mit mehr als 5 ha besaßen $92 \%$ der Fläche. Das nicht persönlich bewirtschaftete Eigentum, das unter verschiedenen Pachtverhältnissen genutzt wurde und in Peru unter den Begriffen ,feudatario' und ,arrendatario' zusammengefaßt wird, machte $26 \%$ der Produktionseinheiten (23\% der Fläche) aus.

Entsprechend den Zielen der Agrarreform galt es, die Eigentums- und Besitzverhältnissse durch die Enteignung des nicht persönlich bewirtschafteten Eigentums und der großen direkt geführten Besitzungen sowie durch die Abschaffung der Pachtund Arbeiterpachtsysteme zu verändern. Entsprechend der aufgezeigten Eigentumskonzentration wurde auf diese Weise das Latifundiensystem attackiert. Wie aus den Ausführungen über den Stand der Enteignungen und Zuweisungen hervorgeht, wurden bis zum 30. 6. 1976 mit rund 7,8 Millionen ha etwa $44 \%$ der Nutzfläche von 1961 enteignet. Mit 6,6 Millionen ha wurden $88 \%$ an kooperative und präkooperative Produktionsformen übereignet. Das heißt $34 \%$ der Nutzfläche gingen in Eigentum der Landarbeiter und, feudatarios' über. Eingerechnet die Comunidades Campesinas steigt der Anteil auf 37\%. Aus der gesetzlichen Grundlage der Agrarreform und den Enteignungs- und Zuweisungszahlen wird deutlich, daß sich die Eigentums- und Besitzverhältnisse de jure, aber wohl auch überwiegend de facto, drastisch verändert haben. Aus den Ergebnissen des Zensus von $1972^{20}$ geht über die Transformation der Agrarstruktur hervor:

- Es wird praktisch kein Privateigentum in Form von juristischen Gesellschaften ausgewiesen.

- Die Pächter (1961: 10\% der Einheiten und 12,8 \% der Fläche) verringerten sich mit $4 \%$ resp. $3,7 \%$ entscheidend.

- Die Arbeiterprachtsysteme konnten ebenfalls abgebaut werden, wenn auch nur bedeutsam in ihrem relativen Flächenanteil. Der geringe Rückgang bis 1972 ergibt sich aus der Tatsache, daß bis zu diesem Zeitpunkt nur relativ wenige der traditionellen Hazienden, zu deren konstituierenden Merkmalen derartige präkapitalistische Arbeitsverhältnisse gehören, angetastet wurden.

- 1972 besaßen CAP und SAIS zwar erst 14,5\% der Fläche. Ihr Anteil stieg jedoch bis zum 30. 6. 1976 auf $28 \%$ und läßt klar ihre Priorität erkennen.

- Die Comunidades Campesinas haben ihren Landbesitz durch Zuweisungen ebenfalls vergrößern können (1961: 10,1 \% der Gesamtfläche, 1972: 26 \%).

- Die direkt bewirtschafteten bäuerlichen Einzelbetriebe haben sich um 4\% in der Vergleichsperiode erhöht. Der Flächenanteil hat sich durch die Enteignung der Großbetriebe um 10\% verringert.

Zusammenfassend ist festzustellen, daß sich im Zensus von 1972 schon die neue Eigentumsstruktur im Agrarsektor abzeichnet: neben kollektiven Bewirtschaftungsformen bleibt kleines und mittleres Eigentum bestehen; Pachtverhältnisse auf der Basis von Geld oder Arbeit sind praktisch verboten. Allerdings zeigt der Zensus auch, daß das Minifundienproblem in voller Größe weiter existiert. An dieser Tatsache hat sich auch bis 1976 nichts geändert.

20 Oficina Nacional de Estadística y Censos (ONEC), II Censo Nacional Agropecuario, Resultados Definitivos, Nivel Nacional, Lima 1975, S. 1, S. 13 bis 14 
Betrachtet man die Größenstruktur bzw. die Flächenkonzentration in der peruanischen Landwirtschaft - damit eng verbunden ist das Problem einer effizienten technisch-ökonomischen Größe, aber auch einer die Partizipation erlaubenden organisatorisch und sozial adäquaten Dimension der Unternehmung - , dann ist aus dem Zensusvergleich 1961 mit 1972 als wesentlichste Tatsache festzustellen, daß sich die Zahl der Produktionseinheiten unter 0,5 ha von 157475 auf 336695 erhöht hat. Ahnlich dramatische Ziffern finden sich in allen Größenklassen bis 5 ha. Insgesamt hatten diese Größenklassen eine Zunahme von rund 699000 auf 1830000 Einheiten zu verzeichnen. Der Zuwachs an Einheiten in den anderen Größenklassen (nur in den Einheiten über 2500 ha vollzog sich eine leichte Abnahme) deutet auf eine abnehmende Konzentration hin, die etwas stärker in den relativen Flächenanteilen der Größenklassen sichtbar wird. Mit der Zunahme der Produktionseinheiten bei gleichbleibender Fläche verschärfte sich die Parzellierung. Außerdem verringerte sich die Durchschnittsgröße pro Einheit von 21 ha (1961) auf 17 ha (1972). Als Ergebnis ist festzuhalten, daß die Agrarreform zwar die Eigentumsstruktur stark verändert hat, daß aber die Größenstruktur in ihrem oberen Konzentrationsteil relativ unverändert geblieben ist. Dagegen hat bei den kleinen Produktionseinheiten bis 1972 die Atomisierung erheblich zugenommen. Diese Entwicklung dürfte sich überwiegend vor 1969 vollzogen haben. Der Zensus von 1972 gibt auch keine Auskunft darüber, ob nicht die Flächenkonzentration in der obersten Betriebsgrößenklasse durch die Agrarreform zum Teil gestiegen ist. Die Kenntnis von Einzelbeispielen läßt zumindest die Aussage zu, daß in einigen Fällen wesentlich größere Unternehmenseinheiten geschaffen wurden. Große Schwierigkeiten in der Führung einiger Unternehmen haben dazu geführt, daß an ihrer Teilung und Restrukturierung gearbeitet wird. Nach Informationen des Agrarministeriums waren im Juni 1976 etwa 120 Unternehmen für "Restrukturierung und Anpassung“ vorgesehen. Dabei bleibt allerdings offen, ob es sich neben Unternehmensteilungen nicht auch um Reprivatisierungen durch Landaufteilung oder sogar Rückgabe an frühere Eigentümer handelt ${ }^{21}$.

\section{Auswirkungen auf die Arbeitsplatzschaffung}

Ein wichtiger Stellenwert in der Beurteilung der Agrarreform kommt auch ihrem Einfluß auf die Beschäftigungssituation in ländlichen Gebieten zu, ausgedrückt z. B. in der Zahl der begünstigten Personen bzw. der zusätzlich geschaffenen Arbeitsplätze, die ein angemessenes Einkommen sichern.

Ein Vergleich der Zensuszahlen von 1961 und 197222 über das demographische Wachstum in Peru ergibt u. a.:

- Die ländliche erwerbstätige Bevölkerung stagniert bei rund 1,5 Millionen.

— Wenn auch die ländliche Bevölkerung zwischen 1961 und 1972 nur um 0,5 \% p. a. zugenommen hat, die städtische Bevölkerung dagegen durch die immer stärker werdende Migration um 5\% p. a. gewachsen ist, stellt der land- und

21 Vgl. Hardtko, A. C., Revolution im Rückwärtsgang, in: Frankfurter Rundschau, 5. 1. 1977.

22 Oficina Nacional de Estadística y Censos, Censos Nacionales: VII de Población, II de Vivienda, 4 de Junio de 1972, Resultados Definitivos, Nivel Nacional, Lima, Agosto 1974, S. 745 ff.; ONEC, Boletín de Análisis Demográfico Nr. 16, Perspectivas de crecimiento de la población del Perú 1960-2000, Lima, Diciembre 1975, S. 4 ff. 
forstwirtschaftliche Sektor mit $40 \%$ der erwerbstätigen Bevölkerung (1961: 49,1\%) noch immer die meisten Arbeitsplätze.

- Der in der Land- und Forstwirtschaft beschäftigte Teil der ländlichen erwerbstätigen Bevölkerung belief sich 1972 auf etwa 83\%. Der Prozentsatz variierte gegenüber 1961 kaum, d. h. die ländliche Produktionsstruktur blieb relativ unverändert.

Um die Land-Stadt-Disproportinalitäten zu verringern, den Migrationsstrom abzubremsen und den hohen Prozentsatz an Unterbeschäftigten (1974 über 65\% der ländlichen erwerbstätigen Bevölkerung) abzubauen, müßten die Arbeitsplätze pro Jahr im Vergleich zum Wachstum der ländlichen Erwerbstätigen überproportional zunehmen. Die direkten und indirekten Effekte der Agrarreform auf die Beschäftigungssituation in den ländlichen Gebieten sind anfänglich vielfach $-z$. T. auch bewußt aus propagandistischen Gründen - überschätzt worden. Das gilt vor allem für die Schaffung von Arbeitsplätzen in der Landwirtschaft.

Geht man davon aus, daß von der Agrarreform 400000 Familien ${ }^{23}$ begünstigt werden sollen und nimmt pro Familie eine begünstigte Person an, dann würden nur $27 \%$ der erwerbstätigen ländlichen Bevölkerung erfaßt, an die etwa 49,6\% der Flächen für Ackerbau und künstliche Weiden sowie 42,4 \% des natürlichen Weidelandes übertragen werden sollen ${ }^{24}$. Bis zum 30. 6. 1976 konnten erst 18,7\% der ländlichen Bevölkerung begünstigt werden.

In den offiziellen Angaben über die Agrarreform wird mit der Zahl der begünstig. ten Familien gearbeitet. Zum Begriff „begünstigt" (spanisch: beneficiario) ist anzumerken, daß auch die Bauern darunter fallen, die mit 3 ha nur die sog. unidad agrícola familiar besitzen. Agrarexperten haben für Peru ausgerechnet, daß diese Größe für die Sicherung des Subsistenzminimums nicht ausreicht. Unter die sog. beneficiarios werden auch die Beschäftigten der neuen Unternehmen gerechnet, die keinen festen Arbeitsplatz haben. Ebenso sind die Mitglieder der Comunidades Campesinas, die einer SAIS angehören, bei diesen Angaben eingeschlossen, obwohl sie überwiegend in der SAIS keine Arbeit finden und ihr Gewinnanteil - vielfach nur über Gemeinschaftsprojekte realisiert - sehr reduziert ist. Bei einer strikteren Auslegung des Begriffs „beneficiario“ ist die Anzahl der durch die Agrarreform begünstigten Personen noch geringer. Als Nutznießer werden begünstigte Familien aufgeführt. Dieser Begriff verleitet zunächst dazu, die Anzahl z. B. mit der durchschnittlichen Zahl der Familienmitglieder zu multiplizieren. Die Realität sieht jedoch anders aus: durch die Agrarreform wird nicht genügend Boden zugewiesen, um einen Familienbetrieb zu gründen, der allen Mitgliedern ausreichend Arbeit und Einkommen garantiert. Im Fall der Mitglieder von CAP oder SAIS hat nur eine Person, höchstens zwei, einen festen Arbeitsplatz. Würden die Agrarreformangaben nur diejenigen Personen umfassen, die einen festen Arbeitsplatz und damit ein gesichertes Einkommen durch die Agrarreform bekommen haben, sänken die Angaben weiter. Um keine großen Irrtümer aufkommen zu lassen, müßten die Nutznießer zumindest an der Zahl der begünstigten Personen gemessen werden.

23 Angaben des ehemaligen Landwirtschaftsministers Valdez Angulo am 16. 5. 1974, in: Valdez Angulo, E., El sector agrario..., a. a. O., S. 25; Horton gibt nach Informationen der DGRAAR die begünstigten Familien mit 340000 an (Horton, D., Land Reform and Reform Enterprises ..., a. a. O., S. 79); Figueroa, A., Estudio por países sobre el empleo rural: Perú, OIT, Ginebra 1976, S. 159, geht von 342000 Familien aus.

24 Angaben bei Horton, D., Agrarian Reform and Reform Enterprises ... a. a. O., S. 82. 
D. L. 17716 legt fest, daß Zuweisungen an die Teile der ländlichen Bevölkerung erfolgen sollen, die kein oder zu wenig Land besitzen. Dazu sind die Landarbeiter (1961: 450 000; 1972: 325 000) und die Kleinstbauern (1961: 700 000; 1972: 1,1 Millionen $)^{25}$ mit bis zu 5 ha zu rechnen. Von der Agrarreform müßten also 1,4 Millionen landarme und landlose Bauern begünstigt werden. Entsprechend den Planzahlen der DGRAAR können jedoch nur zwischen $23 \%$ und $27 \%$ dieser Gruppe, d. h. etwa ein Viertel der marginalisierten ländlichen Bevölkerung, von der Agrarreform erreicht werden. Bis zum 30. 6. 1976 wurden rund $82 \%$ dieses Solls erfüllt. Nach Angaben der DGRAAR sind die ehemaligen Landarbeiter - in geringerem Maße die sog. feudatarios - der Hazienden vor allem der Costa, aber auch der Sierra und Selva die von der Agrarreform am stärksten favorisierte Gruppe: bis zum 30. 5. 1975 rund 108000 Arbeitnehmer von Genossenschaften und 24000 von den SAIS'6. Das bedeutet, daß 1975 etwa 132000 begünstigten Lohnabhängigen etwa 270000 nichtbegünstigte Lohnabhängige gegenüberstanden. Weiterhin sind rund 1 Million Minifundistas $(<5 \mathrm{ha}$ ) ausgeschlossen. Diese Verteilung zeigt, daß die marginalisierte Masse der ländlichen Bevölkerung von der Neuordnung der Eigentumsstruktur nicht profitiert hat, sondern soziale Gruppen Nutzen daraus gezogen haben, die zum Teil schon vor der Agrarreform zu den relativ privilegierten Einkommensbeziehern gehörten. Hätte man jedem Bauern ohne oder mit nicht ausreichendem Land die sog. unidad agrícola familiar zuweisen wollen, so hätte man mit rund 2,1 Millionen ha Land mehr als 50\% der 1972 für Ackerbau und künstliche Weiden genutzten Fläche übereignen müssen.

Ursachen für die begrenzte Reichweite der Agrarreform sind die relative Knappheit an Boden, vor allem für intensive Nutzung, die Durchsetzung von möglichst effizienten Unternehmensheiten und damit der Verzicht auf die Aufteilung der landwirtschaftlichen Großbetriebe sowie die Erhaltung des Mittelbesitzes. Bewässerungs-, Siedlungs- und Landrückgewinnungsprojekten als ergänzenden Maßnahmen der Agrarreform kommen entscheidende Bedeutung zu.

Durch die Agrarreform erhält also nur ein Teil der Landarbeiter und Kleinbauern als Mitglieder von CAP und SAIS oder durch die Gründung von bäuerlichen Einzelwirtschaften Land. Der weitaus größere Teil der erwerbstätigen Bevölkerung muß permanent oder saisonal Arbeit als Tagelöhner bei Mittel- und Großbetrieben suchen. Die Zahl der als Tagelöhner beschäftigten Wander- oder Erntearbeiter (ein geringer Teil von ihnen ist in den Agrarreformunternehmen fest beschäftigt, ohne Mitglied zu sein) hat sich seit Bildung der Genossenschaften erhöht. Die Genossenschaftsmitglieder versuchen ihre Gewinn- und Sozialleistungsanteile zu maximieren, indem sie keine neuen Mitglieder aufnehmen bzw. versuchen, den festen Stamm der Arbeitnehmer der Ex-Hazienda zu reduzieren. Roca ${ }^{27}$ stellte für die acht Zuckergenossenschaften fest, daß sich der Anteil der sog. eventuales von 11,5\% (1968) auf 14,8\% (1972) der Belegschaft erhöht hatte. Von den Mitgliedern verringerte sich der Anteil der festangestellten Arbeiter von 77,4\% auf 70,1\%. $4 \%$ entfielen auf eine Mitgliedererhöhung im Angestelltenverhältnis! Ähnliche Tendenzen sind von anderen Genossenschaften bekannt. Die sog. eventuales, die nicht am Gewinn und nur zum Teil an den Sozialleistungen partizipieren, haben,

25 INP, Dirección Nacional de Estadística y Censos, Primer Censo Nacional Agropecuario. . . a. a. O., S. 11; ONEC, II Censo Nacional Agropecuario, .... a. a. O., S. XV und S. 12.

26 Figueroa, A., Estudio por países ..., a. a. O., S. 161.

27 Roca, S., Las cooperativas azucareras del Perú - Distribución de ingresos, ESAN, Lima 1975, S. 46 ff. 
abgesehen von einem nicht stabilen Beschäftigungsverhältnis, ein niedrigeres Einkommen als die Mitglieder. In den Zuckergenossenschaften stieg das durchschnittliche Lohnniveau der Arbeiter (Mitglieder) von 1968 bis 1972 um 134\%, das der sog. eventuales nur um $57 \%$ \% Unternehmungen, die Denunzierungen in der Offentlichkeit über die Freisetzung von Beschäftigten sowie das Inkrafttreten von D. L. 21334 (10. 12. 1975), in dem festgelegt wird, daß in jedem Unternehmen mit über 9 ha kultivierbarem Land die Relation 5:1 von bewässertem Land (in ha) zu festangestellten Arbeitern gewahrt sein muß, zwingt einige Genossenschaften zumindest zur Eingliederung der sog. eventuales permanentes. Figueroa kalkuliert, daß bei kontrollierter Handhabung des Gesetzes etwa 120000 Arbeitsplätze geschaffen werden könnten ${ }^{29}$. Das würde ein stabiles Auskommen für $44 \%$ der nicht begünstigten Landarbeiter bedeuten.

Der relativ geringe Beschäftigungseffekt der Agrarreform wurde von der peruanischen Regierung gegenüber der Öffentlichkeit ab 1974 offen zugegeben. Gleichzeitig propagierte man ab 1976 im Rahmen des Projektes GEAR (Generación del Empleo en el Ambito Rural ${ }^{30}$ ) die Schaffung zusätzlicher Arbeitsplätze durch die Diversifizierung der ländlichen Produktionsstruktur der Sierra, vor allem der sog. áreas deprimidas im Süden, auf der Grundlage der PIAR ${ }^{31}$. Ziel des Projektes ist, neue Arbeitsplätze durch Investitionen in Handwerk und Kleinindustrie sowie im Dienstleistungsbereich für die neuen Unternehmen zu schaffen. Erster Schritt dieses Programms ist die Identifizierung von möglichst arbeitsintensiven Projektideen durch multidisziplinäre Gruppen, die ihre Arbeit im November 1976 in den am wenigsten entwickelten Distrikten Piuras, Cajamarcas und Cuzcos begonnen haben $^{32}$. Wie weit die prekäre Haushaltslage, der Druck der städtischen Massen und abweichende Entwicklungsprioritäten der Regierung dieses Programm zu einer Propagandakampagne degradieren, bleibt abzuwarten.

\section{Einkommenseffekt der Agrarreform}

Die Veränderung der Eigentumsstruktur hat zu Einkommenserhöhungen und Einkommensnivellierungen zwischen den Beschäftigtengruppen - soweit es sich um Mitglieder handelt - der durch die Agrarreform begünstigten Unternehmen geführt. Bei der Beurteilung der Auswirkungen der Agrarreform auf Einkommenshöhe und -verteilung muß ein direkter und ein indirekter Distributionseffekt berücksichtigt werden. Der direkte Effekt betrifft die begünstigten Personen, die ihr Einkommensniveau durch Lohn- oder Gehaltssteigerungen, durch Beteiligungen am Gewinn, durch bessere Sozialleistungen und in ganz seltenen Fällen auch durch höhere Nebeneinkünfte (z. B. eigengenutzte Parzelle) verbessern konnten. Der indirekte Effekt zeigt sich bei den nichtlandwirtschaftlichen Erwerbsmöglichkeiten, in denen durch eine Erhöhung der Ausgaben der landwirtschaftlichen Erwerbs-

\footnotetext{
28 Roca, Santiago, Las cooperativas azucareras del Perú ..., a. a. O., S. 45 und 46.

29 Figueroa, A., Estudio por países ..., a. a. O., S. 167.

$30 \mathrm{La}$ Prensa, 23. 8. 1976. die dazu dienen, die von der Agrarreform begünstigten Personen in einem abgegrenzten Gebiet so zu organisieren, daß sie eine sozioökonomische Einheit bilden. Der PIAR dient der Abstimmung und Neuordnung der Produktionsseite, wie der wirtschaftlichen und sozialen Förderung der Mitglieder (vgl. CENCIRA, Manual de adjudicaciones, Lima, Febrero 1972, S. 43). Das bedeutet, daß der PIAR seiner Konzeption nach mehr sein soll als eine Landreform nach Regionen.

32 La Prensa, 27. 10. 1976.
} 
tätigen zusätzliche Einnahmen bzw. Überschüsse geschaffen werden. Allerdings wird der indirekte Einkommenseffekt aufgrund der derzeitigen Wirtschafts- und Sozialstrukturen vor allem städtischen Gruppen (z. B. Zwischenhändler) zugute kommen. Der Einkommenstransfer vom Land zur Stadt dürfte sich dadurch vergrößert haben, aber vor allem durch die Zahlungen der Agrarschuld durch den Staat an die Exeigentümer und durch die begünstigten Unternehmen und Personen an den Staat.

Über die Einkommensverteilung in Peru liegen die Untersuchung von Webb für $1961^{33}$ und die der Encuesta Nacional de Consumo de Alimentos (ENCA) für 1972 vor $^{34}$. Beide Studien kommen für unterschiedliche Zeitpunkte zu dem Ergebnis, daß die Relation durchschnittliches Einkommen zu durchschnittlichem ländlichen Einkommen 2:1 ist. Mittels der Angaben von ENCA lassen sich außerdem einige Aussagen zur Einkommensstruktur der ländlichen Familie 1971/72 machen.

Tabelle 6 Durchschnittliches Einkommen pro Familie und Anteil der ländlichen Familien nach Regionen 1971/72

\begin{tabular}{lcccc}
\hline Region & \multicolumn{2}{c}{ Ländliche Gebiete } & \multicolumn{2}{c}{ Peru } \\
& $\begin{array}{c}\text { Einkommen } \\
\text { (S/. pro Jahr) }\end{array}$ & $\begin{array}{c}\text { Familien } \\
\text { (in } \%)\end{array}$ & $\begin{array}{c}\text { Einkommen } \\
\text { (S/. pro Jahr) }\end{array}$ & $\begin{array}{c}\text { Familien } \\
\text { (in \%) }\end{array}$ \\
\hline Costa & $37540(1,7)$ & 7,5 & $87120(1,7)$ & 40,6 \\
Sierra & $17420(0,8)$ & 40,4 & $24080(0,5)$ & 50,6 \\
Selva & $33800(1,5)$ & 6,5 & $41500(0,8)$ & 8,6 \\
Peru & $22150(1,0)$ & 54,4 & $51170(1,0)$ & 100,0 \\
\hline
\end{tabular}

Anmerkung: Die Zahlen in Klammern sind Indexwerte.

Quelle: Encuesta Nacional de Consumo de Alimentos (ENCA), Convenio MEFENCA, Niveles de ingreso de las familias en el Perú, Lima, Abril 1975, Tabelle 2, S. 22, und Tabelle 4, S. 25.

In der Einkommensstruktur sind deutliche Disparitäten zwischen dem durchschnittlichen nationalen Einkommen pro Familie und dem ländlichen Familieneinkommen zu erkennen, das nur 43,3\% des nationalen Durchschnitts erreicht. Die entsprechende Relation beträgt in der Küstenregion 43,1\%, in der Sierra 72,3\% und in der Selva $76 \%$. In den beiden zuletzt genannten Regionen existieren nur wenige städtische Gebiete mit relativ höherem Einkommen. Innerhalb der ländlichen Einkommen pro Familie bestehen ebenfalls starke Unterschiede. Das Familieneinkommen der Sierra liegt bei $79 \%$ des ländlichen Durchschnitts, in der Costa und Selva dagegen bei $170 \%$ resp. $153 \%$. Das durchschnittliche ländliche Einkommen der Costa erreicht 73,4\%, der Sierra 34,0\% (!) und der Selva 66,1\% des durchschnittlichen Familieneinkommens in Peru.

33 Webb, R., Trends in Real Income in Peru, 1950-1966, Research Program in Economic Development, Discussion Paper No. 41, Princeton, February 1974

34 Dirección General de Asuntos Financieros, Ministerio de Economía y Finanzas, Encuesta Nacional de Consumo de Alimentos (ENCA), Niveles de ingreso de las familias en el Perú, Lima, Abril 1975. 
Der Sample von ENCA bestätigt die bekannte Tatsache, daß die ländliche Sierra das ärmste Gebiet Perus ist und daß innerhalb dieser Region wiederum das südliche Gebiet, Teil der sog. mancha india ${ }^{35}$, mit $74 \%$ des durchschnittlichen ländlichen Einkommens das relativ unterentwickeltste ist ${ }^{36}$. Zahlreiche Fallstudien kommen zu dem Ergebnis, daß in jeder Region, jeder Subregion bis hin zu den Mikroregionen wie z. B. einer Comunidad Campesina erhebliche Einkommensunterschiede bestehen ${ }^{37}$. Entsprechendes gilt in etwas abgemilderter Form für Costa und Selva, wo z. B. zwischen und in den Genossenschaften merkliche Niveauunterschiede im Einkommen bestehen ${ }^{38}$. Auch Webb stellt in seiner Studie heraus, daß ein wesentliches Charakteristikum der Einkommensentwicklung zwischen 1950 und 1966 die zunehmende Ungleichheit der Einkommensverteilung bei den Kleinbauern ist ${ }^{39}$.

Für 1961 kalkuliert Webb ${ }^{40}$, daß das durchschnittliche Einkommen des modernen Sektors (Unternehmen mit mehr als fünf Beschäftigten, Zuckerhazienden, öffentlicher Sektor) fast viermal so hoch war wie das des traditionellen ländlichen Sektors (Lohnarbeiter, kleine und mittlere Bauern bis 50 ha). Dieser Unterschied verstärkte sich; denn das Realeinkommen des traditionellen Sektors nahm in den ländlichen Gebieten zwischen 1950 und 1966 nur um 1,3\% p. a. zu. Im Landesdurchschnitt konnte ein Einkommenszuwachs von $2,1 \%$ p. a. realisiert werden ${ }^{41}$. Die Einkommensdifferenzen zwischen den Gruppen des ländlichen traditionellen Sektors haben sich ebenfalls verschärft. Die Realeinkommen der Lohnabhängigen der Sierra stiegen um 1,5\%, die der Kleinstbauern um $0,8 \%$ und die der kleinen bis mittleren Landbesitzer (5 bis $50 \mathrm{ha}$ ) um 2,7\% p. a. ${ }^{42}$ ). Da die landwirtschaftliche Produktion im Zeitraum 1967 bis 1974 nur einen Zuwachs von 1,4\% p. a. ${ }^{43}$ zu verzeichnen hatte, ist realistisch anzunehmen, daß die Realeinkommen in dieser Periode stagnierten. Wenn also keine oder nur eine geringe Erhöhung des durchschnittlichen ländlichen Realeinkommens seit 1969 stattgefunden hat, dürfte - wie Feder feststellt - im traditionellen ländlichen Sektor aufgrund der Preissteigerungen und der Verschlechterung der Austauschrelationen zwischen ländlichen und städtischen Gebieten die Verarmung zugenommen haben.

Die Höhe des Einkommenseffektes durch die Eigentumsumverteilung im Verlauf der Agrarreform ist aufgrund fehlender statistischer Angaben nur sehr schwer abschätzbar. Van de Wetering kalkuliert ${ }^{44}$, daß, wenn sämtliche Ländereien unter die Agrarreform gefallen wären, etwa $14 \%$ bis $18 \%$ des landwirtschaftlichen Einkommens, d. h. 2,5\% bis 3\% des Volkseinkommens, transferiert würden. Da aber nur etwa $40 \%$ des Bodenbesitzes von der Agrarreform erfaßt worden sind, wurden etwa $1 \%$ bis $2 \%$ des Volkseinkommens umverteilt. Dabei ist zu berücksichtigen, daß der Einkommenstransfer nicht vom obersten Quartil in das unterste stattfindet, sondern z. B. im Fall der Zuckerhazienden innerhalb des obersten, also des reichsten Quartils. Die früheren Arbeitnehmer und heutigen Eigentümer der

\footnotetext{
35 Als sog. mancha india bezeichnet man die Departements mit dem höchsten Anteil an indios: Ancash, Apurímac, Ayacucho, Cuzco, Huancavelica, Puno.

36 Dirección General de Asuntos Financieros, Ministerio de Economía y Finanzas, Encuesta Nacional ..., a. a. O., S. 29.

37 Vgl. z. B. Gaitzsch, A., Die peruanische Agrarreform ..., a. a. O., S. $116 \mathrm{ff}$.

38 Vgl. z. B. Roca, S., Las cooperativas azucareras del Perú ..., a. a. O., S. $72 \mathrm{ff}$

39 Webb, R., Trends in Real Income in Perú, 1950-1966, . . . a. a. O., S. 41.

40 Webb, R., und Adolfo Figueroa, Distribución del ingreso en el Perú, IEP, Perú Problema 14, Lima, noviembre 1975 , S. 75.

41 Webb, R., Trends in Real Income in Perú, 1950-1966, . . a. a. O., S. $41 \mathrm{f}$.

42 Alle Angaben aus Webb, R. und Adolfo Figueroa, Distribución del ingreso en el Perú ..., a. a. O., S. 45.

43 Figueroa, A., Estudio por países ..., a. a. O., S. 57.

44 Webb, R. und Adolfo Figueroa, Distribución del ingreso en el Perú, . . a. a. O., S. 132.
} 
agroindustriellen Komplexe gehörten schon immer zu den höchsten Einkommensbeziehern. An der 60prozentigen Erhöhung der Realeinkommen in den Zuckerkooperativen 1968/72 (Redistributionseffekt und Wachstum) partizipierten die Arbeiter am stärksten, die sog. eventuales am wenigsten ${ }^{45}$. Dadurch verstärkte sich der Einkommensunterschied zwischen diesen beiden Gruppen, während zwischen den Mitgliedern im Angestelltenverhältnis und den Arbeitern eine Nivellierungstendenz einsetzte. Auch die Lohnabhängigen der übrigen Exhazienden der Küste und Selva gehörten einkommensmäßig keineswegs zur Basis der Einkommenspyramide. Auf die einkommensschwächsten Teile Perus, d. h. die Lohnabhängigen, die sog. feudatarios und die Minifundistas der Sierra wurden, abgesehen von wenigen Ausnahmen, die unterkapitalisierten Ländereien mit niedriger Produktivität übertragen. $\mathrm{Zu}$ diesen Ausnahmen gehören drei relativ moderne und kapitalstarke Viehhazienden, die in SAIS umgewandelt wurden. Der Wert einer dieser SAIS liegt z. B. bis zu sechsmal über dem einer typischen SAIS in den armen Zonen $^{46}$. McClintock ${ }^{47}$ stellte in der SAIS Cahuide fest, daß von den Hauptbeschäftigtengruppen zwischen 1969 und 1974 ebenfalls die Arbeiter am stärksten an der Redistribution der Einkommen der Ex-Eigentümer und des Verwalters partizipiert hatten. Ebenso war eine Nivellierung in der Entlohnung der Arbeiter und Angestellten sowie der Techniker und Arbeiter eingetreten. Die Techniker bezogen 1969 eine zwölfmal, 1974 nur noch eine sechsmal so hohe Entlohnung wie die Arbeiter. Der Einkommensunterschied zwischen Mitgliedern und den sog. eventuales wuchs auch in diesem Fall. Gleiche Tendenzen nur auf niedrigerem Einkommensniveau finden sich z. B. auch in einer der ärmsten SAIS im Departement Huancavelica: höhere Einkommen bei sich verschärfenden Einkommensdisparitäten ${ }^{48}$.

Ein Redistributionseffekt zwischen den Exhazienden und den Comunidades Campesinas, wie er mit dem SAIS-Modell geplant ist, vollzieht sich nur in geringem Umfang, da einerseits die Nettoüberschüsse, vor allem wenn die Tilgung der Agrarreformschuld beginnt, gering sind ${ }^{49}$, andererseits die Umverteilung in bar von der SAIS möglichst niedrig gehalten und oft in Infrastrukturprojekten vorgenommen wird, die auch der SAIS Vorteile bringen. Von seiten des Managements z. B. der SAIS Tupac Amaru argumentiert man, daß dem Redistributionseffekt mittels produktiver Investitionen für die Comunidades mehr Gewicht als Barausschüttungen beigemessen wird, da auf diese Weise eher eine den Lebensstandard der Mitglieder erhöhende Verwendung der Überschüsse zu garantieren ist.

Wie Figueroa ${ }^{50}$ feststellt, beschränkt sich die Umverteilung durch die Agrarreform nicht nur auf den einkommensschwächsten Sektor der Wirtschaft, sondern findet auch nur innerhalb der jeweiligen Subsektoren und nicht zwischen ihnen statt. Der größere Teil des durch die Agrarreform an die zwischen $23 \%$ und 27\% der ländlichen Erwerbstätigen umverteilten Einkommens bleibt im modernen Sektor

45 Roca, Santiago, Las cooperativas azucareras del Perú, a. a. O., S. 44 und S. 46

46 McClintock, C., Sharing the Benefits of Cooperative Agricultural Enterprises with Outsiders in Peru: The Political and Economic Impact of SAIS Cahuide, in: Economic Analysis and Workers' Management, Vol. X, No. 3-4, 1975, S. 258.

47 McClintock, C., Sharing the Benefits of Cooperative ..., a. a. O., S. $260 \mathrm{ff}$.

48 Informationen von F. Bartu, der mit CENCIRA eine Studie über die SAIS Huancavelica anfertigte. Promoviert z. Z. über das SAIS-Modell.

49 Im Fall der SAIS Cahuide kalkulierte man 1972, daß unter Annahme gleich hoher Zuweisungen an die Reservefonds sowie eines gleich hohen verteilungsfähigen Úberschusses bei Tilgung der Agrarschuld ab 1977 (10,9 Millionen S/.) die Produktion im Vergleich zu 1972 um 60\% erhöht werden müßte (vgl. Montoya, Rodrigo u. a., La SAIS Cahuide y sus contradicciones, Lima 1974, S. 103).

50 Webb, R. und Adolfo Figueroa, Distribución del ingreso en el Perú, ... a. a. O., S. 134. 
der Landwirtschaft. Fast drei Viertel der Bauern partizipieren nicht an der Einkommensumschichtung. Um längerfristig auch Umverteilungseffekte im einkommensschwächsten Teil der Nutznießer der Agrarreform realisieren zu können, müßten diese Unternehmen ihre Produktivitäts- und Überschußsituation entscheidend verbessern. Dazu wiederum ist die Verstärkung der komplementären Maßnahmen der Agrarreform wie Beratung, Finanzierung und Ausbildung notwendig.

4. Entwicklung der landwirtschaftlichen Produktion

Ein wesentlicher Punkt im Zusammenhang mit der Einkommenserhöhung und -umverteilung durch die Agrarreform ist die Entwicklung der landwirtschaftlichen Produktion, vor allem in den neuen Unternehmen. Auch im Fall der peruanischen Agrarreform wird argumentiert, daß einerseits durch den Strukturveränderungsprozeß, andererseits durch Nebenwirkungen wie z. B. Bremsung der Investitions- und Produktionstätigkeit aufgrund der Unsicherheit für den Kleinund Mittelbesitz die landwirtschaftliche Produktion zurückgegangen und damit u. a. das Nahrungsmitteldefizit gestiegen ist. Steigende Importe oder eine Verschlechterung der Ernährungssituation wären die Folge. Um zu einem stichhaltigeren Urteil zu gelangen, müßte zwischen einem relativ kurzfristigen und einem längerfristigen Produktionsrückgang unterschieden werden. Würde sich ein langfristiges Absinken der Produktion abzeichnen, müßte im Fall der peruanischen Agrarreform und der damit verbundenen Schaffung von neuen Eigentumsformen auch diskutiert werden, ob die Zuteilung prioritär an Genossenschaften und SAIS zu dieser Produktionsabnahme geführt hat. Damit würde die kollektive gegenüber der individuellen Produktionsform in der Landwirtschaft in Frage gestellt. Außerdem müßten die Entwicklungstendenzen bei den nicht unter die Agrarreform gefallenen Unternehmen in die Betrachtung einbezogen werden. Eine weitere Differenzierung muß zwischen den sog. industriellen Kulturen bzw. den Exportprodukten und der dem Inlandskonsum dienenden Nahrungsmittelproduktion vorgenommen werden. Die Outputentwicklung der verschiedenen Produkte müßte im Zusammenhang mit den verschiedenen Unternehmensgrößenklassen, dem Technologiestand, der Vermarktungs-, Beschaffungs- und Preispolitik im Agrarsektor analysiert werden. Zudem müßte der Trend der Produktionsentwicklung in den Jahren vor der Agrarreform entsprechende Berücksichtigung finden.

Für einen Vergleich der Entwicklung der landwirtschaftlichen Produktion nach Unternehmensgrößen, Eigentumsformen und Produkten, der Aufschluß über die Auswirkungen der Agrarreform bzw. der kollektiven Bewirtschaftungsformen auf die Produktion geben könnte, existiert kein entsprechend aufgeschlüsseltes Material. Für eine Globalbetrachtung nach Betriebsgrößen (Schichtung gemäß Hektarzahl) liegt nur das Material aus dem Zensus von 1961 und dem Zensus von 1972 vor. Veränderungen bis zum Stichjahr 1972 kommt aber mehr konjunkturelle Bedeutung $\mathrm{zu}$; außerdem begann erst ab 1972 eine massive Transferierung des Eigentums an die Produktionsgenossenschaften und SAIS. Um den Einfluß der Agrarreform herausarbeiten zu können, müßten Zeitreihen für den Output einzelner Produkte bzw. ihre Hektarflächen gebildet werden. Die jährlichen Angaben des Ministerio de Agricultura können aufgrund der sehr angreifbaren Schätztechnik höchstens Anhaltspunkte für die Gesamtentwicklung einzelner Produkte liefern. 
Aus diesem Grunde soll an dieser Stelle auch nicht versucht werden, einen Genauigkeit vortäuschenden Zahlenvergleich vorzunehmen, sondern die nachfolgenden Ausführungen beschränken sich darauf, einige Einzelbeobachtungen und Tendenzen aufzuzeigen, die für eine Urteilsbildung über die Problematik der Produktionsentwicklung als wichtig erachtet werden:

- Zwischen 1961 und 1972 wurden nur die natürlichen Weideflächen ausgedehnt. Die Ackerfläche blieb konstant. In den Betriebsgrößenklassen vor allem bis 10 ha hat die Atomisierung und Zersplitterung der Anbauflächen stark zugenommen, d. h. ein weiteres "Abrutschen" von Betriebseinheiten in Produktion nur für den Eigenbedarf sowie eine Zunahme unrationeller Bewirtschaftung sind zu befürchten.

- Die Produktionsausweitung ${ }^{51}$ der letzten 25 Jahre ist in der Landwirtschaft mit 2,9\% p. a. wesentlich geringer gewesen als das durchschnittliche Wachstum des Bruttosozialproduktes (5,5\% p. a.). Der Anteil des landwirtschaftlichen Sektors am Bruttosozialprodukt hat nicht nur relativ an Bedeutung verloren, sondern es gibt deutliche Anzeichen für eine Stagnation. Betrug die Wachstumsrate von 1950 bis 1964 noch durchschnittlich 3,8\% p. a., so lag sie zwischen 1965 und 1974 nur noch bei 1,7\% p. a. Ohne an dieser Stelle auf die analytisch wichtige Differenzierung der Entwicklung von Exportlandwirtschaft und Produktion für den internen Konsum eingehen zu können, sei nur festgestellt, daß die Produktion von Exportprodukten von 1960 bis 1969 eine negative Wachstumsrate von $2,8 \%$ p. a. aufwies. Sie erholte sich von 1970 bis 1974 leicht aufgrund der Erhöhung der Zuckerproduktion. Baumwolle, Wolle und Kaffee hatten weiter rückläufige Produktionsziffern.

- Die peruanische Regierung hat ab 1969 durch direkte und indirekte Lenkung, durch Kontrollen und Interventionen verstärkt in die Wirtschaft, vor allem auch in den Agrarsektor eingegriffen und sich mittels staatlicher Betriebe in steigendem Maße als Unternehmer in Produktion und besonders im Handel betätigt. Allein eine Auflistung der Institutionen ${ }^{52}$ läßt ahnen, wie stark die Entwicklung des Agrarsektors von der staatlichen Bürokratie abhängt; welche Kompetenz- und Koordinierungsprobleme sowie Duplizität der Aktivitäten auftreten müssen; welche Bedeutung der Zahl und Qualifikation der Mitarbeiter sowie den Arbeitsbedingungen im öffentlichen Sektor für die Entwicklung der Landwirtschaft zukommen.

- Die Kreditversorgung des Agrarsektors und die Reorganisation des für die Finanzierung der landwirtschaftlichen Unternehmen fast ausschließlich zuständigen Banco Agrario del Perú weisen bis heute starke Schwächen auf. Entsprechend den gesetzlichen Grundlagen der Agrarreform erfolgt prioritär eine Kreditbedienung der sog. empreses asociativas. Tendenziell wird damit das größte Kreditvolumen in eine reduzierte Gruppe von Exportprodukten und industriellen Kulturen (1972 gingen 60\% der Kredite des Banco de

51 Zahlen stammen aus Figueroa, A., Estudio por países sobre el empleo rural: Perú, a. a. O., S. 53 f.

52 Wichtigste Institutionen zur Durchführung der Agrarpolitik sind der Ministerio de Agricultura mit den regionalen Agrarzonen und den lokalen Agenturen (Abwicklung der Agrarreform, Beratung und Kontrolle der Unternehmen), CENCIRA (Centro Nacional de Capacitación e Investitigación para la Reforma Agraria: Forschung und Ausbildung), Banco Agrario del Perú (Kreditversorgung), EPSA (Empresa Pública de Servicios Agropecuarios: Kommerzialisierung), ENCI (Empresa Nacional de Comercialización de Insumos: Beschaffung von Input), FERTIPERU (Fertilizantes del Perú: Import und Produktion von Düngemitteln), EPCHAP (Empresa Pública de Comercialización de Harina de Pescado: Import von Grundnahrungsmitteln, Export von Baumwolle, Kaffee, Fischmehl). Für Planung, Kontrolle und Preisfestsetzung sind je nach Produkt der Ministerio de Alimentación, de Comercio oder de Industria y Turismo zuständig. 
Fomento Agropecuario in die Finanzierung von Reis und Baumwolle) an der Küste kanalisiert, die vorwiegend von größeren Unternehmen angebaut werden. Die Masse der Bauern, d. h. die Hauptnahrungsmittelproduzenten, sind von der Kreditversorgung ausgeschlossen. An der ungleichgewichtigen Verteilung des Kredites zu Gunsten der Großunternehmen, der Exportprodukte und der industriellen Kulturen an der Küste hat sich durch die Agrarreform kaum etwas geändert.

- Die Intervention des peruanischen Staates in die Agrarpreisbildung und damit in die Entwicklung von Angebot und Nachfrage richtet sich nach der Kategorie der Produkte, dem Selbstversorgungsgrad und der Bedeutung der Produkte für die Versorgung der Bevölkerung. Ab 1972 sieht sich die Regierung verstärkt vor das Problem gestellt, einerseits mittels staatlicher Eingriffe die Preissteigerungen vor allem für Grundnahrungsmittel abzubremsen, andererseits die Produzentenpreise zu erhöhen, um eine Kostendeckung bzw. Überschußbildung - bei steigenden Inputpreisen - in den Unternehmen zu ermöglichen und Anreize zur Produktionssteigerung sowie zur Eingliederung marginaler Flächen zu bieten.

- Die Intervention des Staates in die Preisbildung dient jedoch nicht zuletzt aufgrund des politischen Drucks der städtischen Massen der Kontrolle und Bremsung der Konsumentenpreise. Die unter der Militärregierung zum Teil verschärfte Diskriminierung der Erzeugerpreise hat zur Stagnation und zum teilweisen Rückgang der Produktion beigetragen. Die Importe von Nahrungsmitteln mußten zum Teil gesteigert werden. Bei gleichzeitiger Anwendung eines selektiven Systems der Subventionierung muß der Staat steigende Verluste der staatlichen Unternehmen, in diesem Fall von EPSA, absorbieren. $\mathrm{Da}$ EPSA die in der Regel zu höheren Weltmarktpreisen importierten Produkte $\mathrm{zu}$ den niedrigeren Inlandspreisen abgibt, geht die Subventionierung letztlich zu Lasten der einheimischen Produzenten, die ihre Preisvorstellungen gegenüber diesen Konkurrenzprodukten nicht durchsetzen können. Figueroa ${ }^{53}$ stellt heraus, daß die peruanische Landwirtschaft aufgrund dieser Preispolitik 1973 fas 43 Millionen US-\$ allein bei Weizen und Fleisch verloren hat. Die Preisdifferenz wurde durch die künstliche Überbewertung des Solkurses verstärkt. Dadurch kam es bei den Genossenschaften und SAIS, die Exportprodukte (Zucker, Kaffee, Wolle, Baumwolle) anbauen, ebenfalls zu Einnahmeeinbußen. Das Argument, daß große Anteile der Importsubventionen über den Konsum in die ländlichen Gebiete zurückgehen, konnte durch eine Studie des Ministerio de Economía y Finanzas widerlegt werden ${ }^{54}$ : von 6,5 Milliarden S/. an Subventionen (vor allem Benzin, Reis, Weißbrot, Nudeln, Speiseöl) im Jahre 1975 kamen nur 18\% der ländlichen Bevölkerung zugute. Die Preis- und Subventionspolitik von landwirtschaftlichen Produkten orientiert sich bis heute überwiegend am soziopolitischen Druck der städtischen Konsumenten. Ihr liegt kein förderungspolitisches Konzept, das auch die landwirtschaftlichen Produzenten berücksichtigt, zugrunde. Diese erzielen vielfach nur kostendeckende Preise oder müssen unter Kosten verkaufen, vor allem wenn die Kapitalbedienungs- und Abschreibungskosten angesetzt werden.

53 Vgl. Figueroa, A., Estudio por países sobre el empleo rural: Perú, a. a. O., S. 213.

54 Ministerio de Economía y Finanzas-Encuesta Nacional de Consumo de Alimentos (ENCA), Incidencia de los subsidios en los ingresos de las familias en el Perú: situación actual, Lima, mayo de 1975, S. 1, zitiert nach Figueroa, A., Estudio por paísas sobre el empleo rural: Peru, a. a. O., S. 215. 
- Den niedrigen Erzeugerpreisen standen in den letzten Jahren stark steigende Preise für landwirtschaftlichen Input gegenüber ${ }^{55}$. Die Preise z. B. für Düngemittel - ab 1975 subventioniert - verdrei- bis vervierfachten sich je nach Produkt zwischen 1971 und 1975. Entsprechendes gilt für Schädlingsbekämpfungsmittel und Veterinärprodukte. Die Preise für Saatgut verdoppelten sich zum Teil. Nominallöhne und -gehälter zeigten - wie schon bei den Ausführungen über den Einkommenseffekt angedeutet - überwiegend eine ansteigende Tendenz. Stellt man dazu die Versorgungs- und Verteilungsprobleme beim landwirtschaftlichen Input in Rechnung, wird klar, welchen Schwierigkeiten sich die Unternehmen bei der Produktivitäts- und Produktionsverbesserung allein aus diesem Grund gegenüber sehen.

- Das derzeitige Kommerzialisierungssystem im landwirtschaftlichen Sektor funktioniert noch überwiegend mittels Zwischenhändlern. Die mehr punktuellen Regulierungsmaßnahmen der Regierung durch die Einschaltung staatlicher Unternehmen haben vielfach zu einer weiteren Verzerrung der Absatzsituation beigetragen. Die Abspaltung des Ministerio de Alimentación vom Agrarministerium und der Aufbau des Ministerio de Comercio haben zu Kompetenzund Funktionsteilungen geführt, die sich auf die Durchsetzung einer erzeugeradäquaten Absatz- und Preisgestaltung negativ auswirken. Die bis heute sehr schwache technische Infrastruktur (Silos, Aufkaufstellen, Transportmittel etc.) ist ein weiteres Hemmnis bei der Verbesserung der Absatz- und Beschaffungsmechanismen.

Die Skizzierung dieser Problembereiche, die die Entwicklung des landwirtschaftlichen Sektors entscheidend bestimmen, läßt erkennen, daß für die Beurteilung der Produktionsentwicklung in den Jahren der Agrarreform seit 1969 ein komplexes Beziehungs- und Einflußsystem analysiert werden muß, in dem die Umverteilung des Eigentums und die Schaffung der neuen Unternehmen nur ein wenn auch wichtiges Element ist. Stagnation und teilweiser Rückgang bei einzelnen Produkten sind eine seit langem zu beobachtende Tendenz in der landwirtschaftlichen Produktion Perus. Sie sind weniger auf die Agrarreform zurückzuführen als auf die historisch angelegten heterogenen Produktions- und Sozialstrukturen im Agrarsektor sowie die sie verstärkende Agrarpolitik der die traditionellen Landeliten unterstützenden Regierungen. Um eine Dynamisierung der Produktion - und auch des ländlichen Konsums - einzuleiten, mußten die extensiv bewirtschafteten Großbetriebe enteignet, die präkapitalistischen Abhängigkeitsverhältnisse aufgelöst und die Einkommenskonzentration durch eine breitere Verteilung des Eigentums vor allem des modernen Sektors der Küste abgebaut werden.

Es besteht kein Zweifel, daß die Produktionsentwicklung insgesamt natürlich durch die legale und illegale Dekapitalisierung der Hazienden — sie begann zum Teil schon 1964 - durch die sich verringernde Produktions- und Investitionstätigkeit der Klein- und Mittelbetriebe, durch die teilweise Verwahrlosung von Ländereien u. a. Rückschläge erlitten hat. Seit Übernahme der Unternehmen durch die Genossenschaften haben Schwierigkeiten im Management, in der Arbeitsorganisierung und -disziplin sowie die Tendenz zur Reduzierung der Arbeitszeit durch die Mitglieder die Produktionsentwicklung zum Teil ebenfalls negativ beeinflußt.

55 Vgl. Ministerio de Alimentación, Dirección General de Informática y Estadística, Doc. No. 5, o. S. 
- Insgesamt ist jedoch das Urteil, das Landreform quasi automatisch mit Produktionsrückgang und Schwierigkeiten in der Nahrungsmittelversorgung gleichsetzt, abzulehnen. In der notwendigen differenzierten Analyse kommt den komplementären Maßnahmen der Agrar- und der allgemeinen Entwicklungspolitik besondere Bedeutung zu.

- Auf dem Hintergrund der skizzierten Probleme und der Analyse des wenigen Datenmaterials kann man davon ausgehen, daß die Produktion in der Landwirtschaft nach dem Einbruch 1971/72 im Durchschnitt nicht zurückgegangen ist, daß sich aber auch keine Erhöhung entsprechend den 2,9\% Bevölkerungszuwachs p. a. in den Jahren der Agrarreform vollzogen hat. Folge dürften nicht nur stagnierende durchschnittliche Realeinkommen bei der Mehrzahl der ländlichen Bevölkerung sein, sondern das Defizit in der landwirtschaftlichen Produktion per capita ist auch gestiegen.

\section{Zusammenfassende Schlußfolgerungen}

Abschließend wäre auf der Grundlage der vorher angeschnittenen Probleme, der Kenntnis weiterer Aspekte der Agrarreform und besonders der Entwicklung der neuen Unternehmen, die in diesem Artikel weitgehend unberücksichtigt bleiben mußten, und der politischen Kursänderung in Peru vor allem seit Mitte 1976 die Frage nach den Perspektiven der Agrarreform zu stellen. Die erste Phase brachte eine teilweise Entmachtung der traditionellen Eliten mittels Eigentums- und Einkommensumverteilungen. In ihr wurden die Grundlagen zu Strukturveränderungen gelegt, die es nun mittels der im Agrarreformgesetz postulierten komplementären Maßnahmen zu konsolidieren gilt. Langfristig läßt sich der Redistributionseffekt nur bei gleichzeitigem Wachstum der neuen Unternehmen sichern und erweitern. Die Eigentumsumverteilung dürfte - nicht zuletzt aufgrund des in den letzten Jahren gewachsenen politischen Bewußtseins der ländlichen Bevölkerung - irreversibel sein, wenn auch gewisse Korrekturen in der Struktur und Größe einer Anzahl von neuen Unternehmen notwendig sind. Die aufgezeigte relativ begrenzte Reichweite der Agrarreform muß in Funktion der von den Militärs aufgestellten Ziele und Entwicklungsstrategien beurteilt werden: durch die Veränderungen in der Eigentums- und Besitzstruktur sollen die für eine fortschreitende importsubstituierende Industrialisierung notwendigen Wachstums- und Entwicklungsimpulse in der Landwirtschaft mobilisiert werden. Die vertikal angelegte Redistributionspolitik, d. h. der Transfer von Einkommen innerhalb eines Sektors oder eines Subsektors entspricht diesem Konzept. Insgesamt findet durch die Eigentumsumverteilung ein relativ großer Einkommenstransfer statt, allerdings für eine sehr begrenzte und schon vorher teilweise relativ privilegierte Schicht. Für die Masse der Bauern besteht kaum ein Chance, gezielten Druck auszuüben, um stärker an den Überschüssen des modernen Sektors zu partizipieren. Webb ${ }^{56}$ stellt fest, daß die Bauern außerdem zur Zeit in Peru noch weniger Macht haben als früher, um sich gegen die laufende Verschlechterung der Austauschrelationen zwischen Land und Stadt, Resultat der Preis- und Importpolitik der Militärregierung, zu wehren.

56 Webb, R. und Adolfo Figueroa, Distribución del ingreso en el Perú, a. a. O., S. 93. 
Für die zweite Phase der Agrarreform, die gemäß D. L. 17716 vor allem die Entwicklungschancen der Minifundien durch Parzellenintegration, Flurbereinigung und Aufbau von Servicegenossenschaften bei gleichzeitiger Schaffung von Arbeitsplätzen in nicht landwirtschaftlichen Bereichen verbessern soll, sind die Realisierungschancen nicht sehr aussichtsreich. Geringe Priorität in Relation zu dem Ziel der Agrarreform, die Grundlagen für eine sich verbreiternde Industrialisierung durch die Konsolidierung der neuen Unternehmen zu schaffen sowie der finanziell und personell sehr große Aufwand für die Restrukturierung der Minifundien lassen ein inoffizielles "Zurückstellen" dieser Phase wahrscheinlich sein. Im Entwurf zum Entwicklungsplan 1977 bis 1980 (Plan Tupac Amaru) steht denn auch lapidar unter Punkt 3 der Agrarreformpolitik "Fortsetzung der Aktionen zur Überwindung der Probleme des Minifundismus". Bis 1976 konnten nur wenige Servicegenossenschaften und nur im Ansatz zwei Pilotprojekte zur Flurbereinigung der Kleinstbauern organisiert werden. $\mathrm{Ob}$ im Rahmen der zweiten Etappe der Agrarreform die Regierung sich des Minifundienproblems annimmt, dürfte entscheidend von der politischen Organisationskraft des Agrarsektors und vor allem dieser Gruppe abhängen. Tendenziell dürfte jedoch die Beurteilung von E. Feder zutreffen, daß die Masse der Bauern die Verlierer der peruanischen Agrarreform sind. 
uncertain. The balance between decentralization of power to peasant associations which might well preserve the status quo and egalitarian measures enforced by the central government remained undetermined, as did that between egalitarianism by redistribution of land and by collective agricultural activity.

Changes in Income, Occupation and Production in the Agrarian Sector caused by the 1969 Peruvian Land Reform

\section{By Mechthild Minkner}

The announcement of the Velasco-government's land reform law (No. 17716) on June 24, 1969, marks the beginning of a new phase in the Peruvian land reform process. According to the basic legislation, the agrarian reform is an integral process and an instrument for the transformation and reorientation of the agrarian structure. In this way, it intends to integrate the marginal groups of the rural population to the national development. At the same time the land reform is the crucial factor in order to promote agricultural development and to channel financial ressources to estimulate the industrialization. In the 1969 reform law the maximum size allowed for private landholdings has been reduced, for example in the coastal zone to 150 ha of irrigated cropland (since 1975, 50 ha), agrarian industrial complexes have been submitted to the reform, and "associative enterprises" characterized by joint ownership and self-management have been implemented as a central element of the new structure. After more than 7 years of land reform, the illusions about the great coverage of the reform mesure are gone, and we can find more than one authorized voice stating that the great mass of poor farmers and rural workers are the losers in the 1969 land reform.

The article deals with some aspects of the influence of the land reform on the land tenure patterns, on the rural income and on occupation, as well as on the development of production. However, the statistical information on the changes occured since 1969 in the agrarian sector is very poor. Therefore, the different problems can only be treated along very broad lines. The sources of data are the existing official reports, some academic research carried out on the subject and the interviews done by myself in Lima and in the country side:

Until June 30, 1976, with the expropiation of about 7,8 Million ha and 11110 properties the government has fulfilled $72 \%$ resp. $76 \%$ of the reform goal. The redistribution was carried out more slowly. The priority of "associative enterprises" in the redistribution process is very clear. Agricultural production cooperatives and the quasi-cooperative form known as SAIS are the greatest beneficiaries of the reform, having received $70 \%$ of the redistributed land. The reform has transformed drastically the land property patterns, by eliminating the property of the agricultural companies, reducing all types of relations based on rent and enlarging substantially the area and the importance of cooperative forms and peasant communities. The land redistribution is not designed to reduce the size of the very large holdings. On the contrary, now if the redistribution program is near to completed, fewer large production units will control a larger share of the country's farmland. But in the small size productive units the number of property 
holders has grown, being this a tendency to less concentration in the size structure of agricultural activity.

The influence of the land reform on the occupational situation is far from being satisfactory. The relative scarcity of land, the maintenance of an economically efficient size of production unit and the consolidation of the existing medium size enterprises are the main reasons to explain why only one quarter of the poor and landless population has benefitted from the agrarian reform. About 270000 wage-earners and 1 Million small minifundia landholders were not reached by the redistribution of land. There is also serious evidence that since the establishment of the cooperatives the number of seasonal workers has increased. The effect on income redistribution has been also very limited. There are calculations that only between $1 \%$ and $2 \%$ of domestic income was redistributed. This would be a considerable effect, if the transference of income had been from the highest to the lowest stratum of the income pyramid. But the land reform has only distributed within a subsector and not between the different subsectors. For instance, the coastal workers, who were also before the reform a group with good earnings, have received the most productive enterprises with the greatest net surplus. To the beneficiaries in the poorest part of Peru, the Southern Sierra, have been transfered the extensive and decapitalized cattle-raising units. In this way, the agrarian reform has deepened the gap between different income groups. The development of production is closely related to the land reform. Most of the adversaries of the land reform, also in the Peruvian case, argue that the land reform provokes a decline in production and that consecuently a deficit of agricultural products arises. At the moment, there are no reliable data on this question in Peru. When analyzing the Peruvian agricultural situation it will become clear that the development of production depends on a very complicate set of factors, amongst which the redistribution of land is only one. The governments' intervention in the sector, the control of prices, the precarious credit situation of the farmers, the conditions of commerzialization and the rapidly increasing prices for agricultural inputs in the last years are some of the veriables heavily affecting the development of production. The stagnation of the Peruvian agricultural production has been visible all over the last 20 years. In recent years, it has been in part a by-product of the land reform, but above all it is the result of a heterogenous productive and social structure.

Summing up, we would say that the Peruvian agrarian reform has tackled successfully the latifundia problem. The minifundia problem is far from being solved, and at the present there are not too many chances to continue the reform program in relation to this aspect.

\section{Promotion of the rural poor in India}

\section{By Theodor BergmanN}

After a brief description of the agrarian situation and the causes of near-stagnation of farm production the paper describes a new program of the central government for the marginal rural social strata. The "green revolution" had left out these masses, which are an important economic and political factor. The new agencies 